\title{
Assessment of pulmonary mechanics and breathing patterns during posturally induced glossoptosis in infants
}

\author{
F Cozzi, M Bonanni, D A Cozzi, P Orfei, S Piacenti
}

\begin{abstract}
Respiratory mechanics were studied in nine infants with glossoptosis-apnoea syndrome to determine whether glossoptosis may account for signs of both inspiratory and expiratory airway obstruction. Airflow, oesophageal pressure, inspiratory and expiratory time $\left(T_{i}\right.$ and $\left.T_{e}\right)$, and inspiratory and expiratory resistance $\left(R_{i}\right.$ and $R_{e}$ ) were measured before and during ventilatory phases characterised by glossoptotic pharyngeal obstruction, induced by turning the infants onto their backs. In addition, an attempt was made to correlate the abnormalities in pulmonary mechanics with the clinical features. During partial glossoptotic pharyngeal obstruction, a significant increase was observed in $T_{e}$ and $R_{e}$ and variable changes in $T_{i}$ and $R_{i}$. During severe obstruction, the infants displayed obstructed inspiratory efforts often associated with stridor, as well as obstructed expiratory efforts often associated with audible grunting and retarded expiratory flow pattern. The expiratory grunt was loudest over the neck and mimicked bronchospasm over the chest. These findings indicate that glossoptotic pharyngeal obstruction induces functional airway obstruction which may affect both inspiration and expiration. Expiratory airway obstruction seems, at least in part, to be due to active braking of expiratory flow.

(Arch Dis Child 1996;74:512-516)
\end{abstract}

Keywords: congenital micrognathia, choanal atresia, oesophageal atresia.

Policlinico Umberto I, University of Rome

'La Sapienza', 00161

Rome, Italy:

Paediatric Surgery

Unit

F Cozzi

M Bonanni

D A Cozzi

S Piacenti

Institute of

Anaesthesiology

P Orfei

Correspondence to:

Dr Francesco Cozzi, Head of

Paediatric Surgery,

Policlinico Umberto I, Viale

Regina Elena 324, 00161

Rome, Italy.

Accepted 21 March 1996 upper airway constricting forces generated by inspiratory efforts. This upper airway instability results in recurrent episodes of functional upper airway obstruction, in part due to relapse of the tongue, referred to as 'glossoptosis' by Robin. ${ }^{5}$ Since sleeping is not an essential prerequisite for the occurrence of obstructive apnoea in infancy, we suggested that in infancy the term glossoptosis-apnoea syndrome should be used instead of sleep-apnoea syndrome. ${ }^{1}$

Glossoptotic pharyngeal obstruction - like any variable extrathoracic obstruction impedes the inspiratory phase of respiration, causing inspiratory dyspnoea. However, some $50 \%$ of infants with glossoptosis-apnoea syndrome surprisingly present with signs not only of inspiratory airway obstruction but also of expiratory airway obstruction, particularly during crying, exertion, or respiratory infections. ${ }^{1-4}$ These episodes are often diagnosed as 'bronchiolitis' or 'asthmatic attacks'.

To determine whether glossoptosis should be considered a cause of both inspiratory and expiratory airway obstruction, investigations were carried out on the pulmonary mechanics during glossoptotic pharyngeal obstruction in nine infants with glossoptosis-apnoea syndrome, attempting to correlate the physiological abnormalities with the clinical features.

\section{Methods}

The criterion for patient selection was the presence of one or more clinical manifestations of glossoptosis-apnoea syndrome in infants with congenital micrognathia, choanal atresia, or oesophageal atresia. The main clinical features of the nine infants studied are summarised in table 1 . Patients Nos 1, 2, and 3 had oesophageal atresia with a lower tracheooesophageal fistula. Patient No 2 had mild respiratory problems and later developed failure to thrive. Patient No 4 had marked congenital micrognathia. Patient No 5 had congenital micrognathia associated with cleft palate. Patient No 6 had hemifacial microsomia with severe hemimandibular hypoplasia. Patients Nos 7, 8, and 9 had bilateral choanal atresia.

Informed consent from the parents was obtained before investigations in all nine infants. Infants Nos 1-3 and 5 were studied during sleeping after feeding. Patients Nos 4 and 6-9, who were scheduled to undergo elective surgery, were studied after administration of ketamine ( $5 \mathrm{mg} / \mathrm{kg}$ intramuscularly) immediately before surgery. Ketamine was used because it does not impair the patency of the upper airway. To obtain glossoptotic pharyngeal obstruction we changed the posture of the infants from lateral to supine position.

The oesophageal pressure $\left(\boldsymbol{P}_{\text {oes }}\right)$ was measured with an $8 \mathrm{~F}$ plastic feeding tube filled with water and kept free of bubbles by flushing 
Table 1 Anthropometric and main clinical features

\begin{tabular}{lllllllllll}
\hline Patient No & 1 & 2 & 3 & 4 & 5 & 6 & 7 & 8 & 9 \\
\hline Age at study (days) & 2 & 2 & 7 & 26 & 10 & 180 & 210 & 180 & 30 \\
Sex & F & F & F & M & F & M & M & F & M \\
Diagnosis & OA & OA & OA & CM & CM & HM & CA & CA & CA \\
Weight at study (kg) & 2.8 & 2.6 & 3.1 & 2.9 & 3.0 & 5.6 & 7.1 & 5.0 & 3.5 \\
Inspiratory retractions & + & + & + & - & + & + & + & + & + \\
Opisthotonous & + & - & + & - & + & - & + & + & + \\
Expiratory wheezing/grunting & + & - & + & - & + & + & + & + & + \\
Reflex apnoea/bradycardia & + & - & + & - & + & + & - & + & - \\
Oropharyngeal dysphagia & - & + & + & + & + & + & + & + & + \\
Vomiting/GOR & + & - & - & - & + & - & + & - & - \\
Failure to thrive & - & + & - & - & - & + & - & + & - \\
Cor pulmonale & - & - & - & - & + & + & - & - & - \\
\hline
\end{tabular}

$\mathrm{OA}=$ oesophageal atresia; $\mathrm{CM}=$ congenital micrognathia; $\mathrm{HM}=$ hemifacial microsomia; $\mathrm{CA}=$ choanal atresia; GOR = gastro-oesophageal reflux.

with $2 \mathrm{ml}$ of degassed water between measurements. The catheter was inserted into the oesophagus through the nose or mouth and connected to a pressure transducer (Statham PM 15 ETC; Gould). The end expiratory pressure during a ventilatory period preceding glossoptotic pharyngeal obstruction was taken as the baseline in each patient.

Air flow signals were obtained from a pneumotachograph (Fleisch) connected to a tight fitting mask. The differential pressure signals from the pneumotachograph were measured with a Validyne transducer (model DP 250; Validyne Engineering). An oscillograph (SE oscillograph 3006/DL; SE Laboratories), at high sensitivity, was used to record all signals. During recording, breathing movements were constantly checked and respiratory sounds monitored by stethoscope auscultation.

We defined as partial glossoptotic pharyngeal obstruction an episode with a significant decrease in mean flow without a significant decrease in mean $P_{\text {oes }}$ ( averaged over 7-10 consecutive breaths) on a trace section in comparison with a trace section taken before glossoptotic pharyngeal obstruction (fig 1). An episode of absent or irregular inspiratory flow, despite increasing mean $P_{\text {oes }}$ (averaged over 5-10 consecutive breaths) was defined as severe glossoptotic pharyngeal obstruction (fig 2). The most frequent pattern of irregular
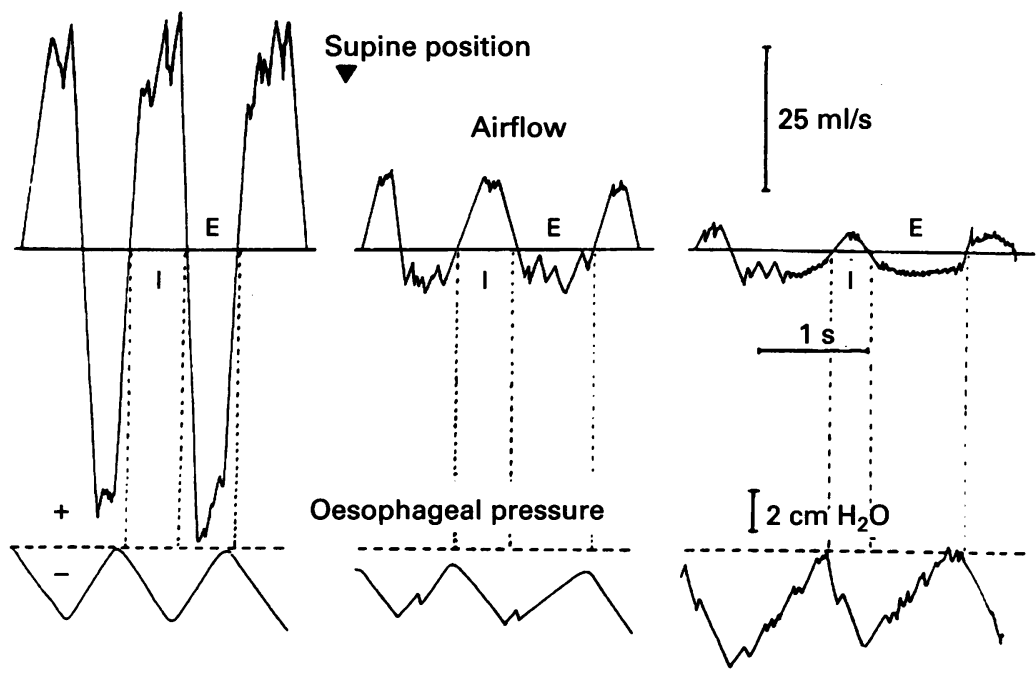

Figure 1. Representative ventilatory phase during partial glossoptotic pharyngeal obstruction. Changing posture from lateral to supine position results in marked decrease in airflow without significant decrease in oesophageal pressure. Note (1) inspiratory time (I) longer than expiratory time (E) in lateral position; (2) progressive prolongation of $E$ and variable changes in I in supine position (redrawn from actual traces). inspiratory flow was similar to that described by Roberts et al in infants with congenital micrognathia, ${ }^{6}$ that is, a sharp reduction in flow during late inspiration (fig 2). During glossoptotic pharyngeal obstruction no attempt was made to validate the $P_{\text {oes }}$ recording by the use of the occlusion test on account of the poor transmission of alveolar pressure to the airway opening.

The inspiratory $\left(T_{i}\right)$ and expiratory $\left(T_{e}\right)$ times were measured on the flow tracing. During absence of flow tracing, the times of inspiratory and expiratory efforts were measured on the $P_{\text {oes }}$ tracing. The mean duration of the respiratory cycle was used as a measure of the respiratory rate $\left(60 / T_{i}+T_{e}\right)$. Airway inspiratory $\left(R_{i}\right)$ and expiratory $\left(R_{e}\right)$ resistance were calculated by the method of Frank et $a l^{7}$ slightly modified. ${ }^{8}$ Mean $T_{i}, T_{e}, R_{i}$, and $R_{e}$ for each patient was calculated manually (over 7-10 breaths) from consecutive inspirations and expirations on sections of the trace taken before and during partial glossoptotic pharyngeal obstruction. Mean $P_{o e s}, T_{i}$, and $T_{e}$ for each of the seven patients with severe glossoptotic pharyngeal obstruction was calculated manually (over 5-10 breaths) from consecutive respiratory attempts on a section of the trace taken during severe glossoptotic pharyngeal obstruction. Data were analysed using Student's paired $t$ test.

\section{Results}

VENTILATORY PERIODS DURING PARTIAL GLOSSOPTOTIC PHARYNGEAL OBSTRUCTION All infants had recurrent episodes of glossoptotic pharyngeal obstruction in the supine position. Episodes of spontaneous obstruction were also observed in the lateral position.

During the episodes of partial glossoptotic pharyngeal obstruction, $T_{i}$ increased significantly in six infants, and decreased or failed to significantly change in three, whereas $T_{e}$ increased significantly in all infants (table 2). Group mean respiratory rate decreased from 65 (SEM 4.5) to 50 (2.8) breaths/min $(\mathrm{p}<0.05)$.

The $R_{i}$ increased significantly in five infants and decreased or showed no significant changes in four, while $R_{e}$ increased significantly in all patients (table 3 ). Group mean $R_{\text {. }}$ increased from 82.6 (12.9) to 171.9 (31.2) $(p<0.03)$, whereas group mean $R_{e}$ increased from 59.3 (12.0) to 184.9 (14.3) $\mathrm{cm} \mathrm{H}_{2} \mathrm{O} /$ litre/s $(p<0.001)$. During partial glossoptotic pharyngeal obstruction, $P_{\text {oes }}$ swings remained in the negative range throughout the entire breathing cycle. Small end expiratory positive pressure swings were detected at the end of the partial obstruction.

VENTILATORY PERIODS DURING SEVERE GLOSSOPTOTIC PHARYNGEAL OBSTRUCTION Seven patients (patients Nos 1, 3, and 5-9) had episodes of severe glossoptotic pharyngeal obstruction. Infants Nos 5 and 6 had episodes of spontaneous severe obstruction even in the lateral position.

In comparison with partial glossoptotic pharyngeal obstruction, during severe obstruction, group mean respiratory rate decreased signifi- 
Table 2 During episodes of partial glossoptotic pharyngeal obstruction (GPO) variable changes occur in inspiratory time whereas a significant increase occurs in expiratory time. During severe GPO both inspiratory time and expiratory time show an increase; values are mean (SEM)

\begin{tabular}{|c|c|c|c|c|c|c|}
\hline \multirow{2}{*}{$\begin{array}{l}\text { Patient } \\
\text { No }\end{array}$} & \multicolumn{3}{|c|}{ Inspiratory time (ms) } & \multicolumn{3}{|c|}{ Expiratory time (ms) } \\
\hline & Before GPO & Partial GPO & Severe GPO & Before GPO & Partial GPO & Severe GPO \\
\hline 1 & $242(13)$ & $616(48) \dagger$ & $909(14) \dagger$ & $428(78)$ & $805(94) \dagger$ & $1554(64) \dagger$ \\
\hline 2 & $532(10)$ & $606(14) \dagger$ & - & $584(21)$ & $748(37) t$ & - \\
\hline 3 & $310(4)$ & $334(6) t$ & $408(59)$ & $418(8)$ & $532(27) t$ & $1216(194)^{\star}$ \\
\hline 4 & $550(13)$ & $432(12) t$ & - & $462(11)$ & $1032(58) \dagger$ & - \\
\hline 5 & $856(16)$ & $576(62) \dagger$ & $824(35) \dagger$ & $448(24)$ & $608(39) t$ & $1984(408) \dagger$ \\
\hline 6 & $445(44)$ & $511(68)$ & $651(28)^{\star}$ & $465(24)$ & $705(103)^{\star}$ & $954(188)$ \\
\hline 7 & $580(52)$ & $518(47)$ & $640(24)$ & $487(25)$ & $693(58)^{\star}$ & $1080(128)^{\star}$ \\
\hline 8 & $408(13)$ & $524(12) \dagger$ & $774(91)^{\star}$ & $448(24)$ & $577(17) \dagger$ & $1476(240) \dagger$ \\
\hline 9 & $500(57)$ & $628(125)^{\star}$ & $951(388)$ & $400(12)$ & $502(50)^{\star}$ & $505(54)$ \\
\hline
\end{tabular}

Comparison between values before and during partial GPO and during partial and severe GPO.

${ }^{\star} \mathrm{p}<0.05 ; \dagger \mathrm{p}<0.01 ; \S \mathrm{p}=\mathrm{NS}$.

cantly from 50 (2.8) to 31 (2.9) breaths $/ \mathrm{min}$ $(p<0.01)$ due to a prolongation of $T_{i}$ and $T_{e}$ (table 2). During these phases with low respiratory rates, all seven patients had inspiratory $P_{\text {oes }}$ swings which were often biphasic and coincided with partially or totally obstructed inspiratory flow (fig 2). Clinically, inspiration was accompanied by severe retractions which were more evident at the jugular incision, and by paradoxical movements of the anterior chest wall, little or no air entry, and often an audible stridor.

During severe glossoptotic pharyngeal obstruction, the expiratory $P_{\text {oes }}$ swings were mainly positive. There was a phasic increase in expiratory $\mathbf{P}_{\text {oes }}$ swings which coincided with a phasic increase in retarded expiratory flow (fig 2). At the end of expiration, the retarded expiratory flow coincided with either a drop in positive $P_{\text {oes }}$ swings for some breaths, or with an increase in positive end expiratory $P_{\text {oes }}$ swings for other breaths (fig 2). Expiration was accompanied by contraction of the anterior abdominal wall muscles and often by audible expiratory grunting which was loudest over the neck and more distant over the chest, where it mimicked bronchospasm.

\section{Discussion}

The most important data emerging from our study during episodes of partial glossoptotic pharyngeal obstruction were: (1) slowing of the breathing rate due mainly to prolongation of $T_{e}$; (2) increase in $R_{e}$; and (3) various modifications in $T_{i}$ and $R_{i}$. In many respects these findings are comparable to those found in children and adults with sleep-apnoea syndrome. During obstructive sleep-hypopnoea preceding obstructive sleep-apnoea, Martin et

Table 3 During episodes of partial glossoptotic pharyngeal obstruction (GPO) inspiratory resistance shows variable changes whereas expiratory resistance shows a significant increase; values are mean (SEM)

\begin{tabular}{|c|c|c|c|c|}
\hline \multirow{2}{*}{$\begin{array}{l}\text { Patient } \\
\text { No }\end{array}$} & \multicolumn{2}{|c|}{ Inspiratory resistance $\left(\mathrm{cm} \mathrm{H}_{2} \mathrm{O} / \mathrm{l} / \mathrm{s}\right)$} & \multicolumn{2}{|c|}{ Expiratory resistance $\left(\mathrm{cm} \mathrm{H}_{2} \mathrm{O} / \mathrm{l} / \mathrm{s}\right)$} \\
\hline & Before GPO & During GPO & Before GPO & During GPO \\
\hline 1 & $103.0(12.6)$ & $377.0(46.7) \dagger$ & $72.0(14.9)$ & $319.0(29.9) \dagger$ \\
\hline 2 & $37.7(5.2)$ & $112.7(9.6) \dagger$ & $46.9(5.3)$ & $167.5(12.9 \dagger)$ \\
\hline 3 & $97.9(8.4)$ & $324.4(30.9) \dagger$ & $104.3(1.8)$ & $197.5(19.3) \dagger$ \\
\hline 4 & $11.2(1.3)$ & $147.5(42) \dagger$ & $19.6(2.8)$ & $204.2(13.3) \dagger$ \\
\hline 5 & $78.9(13.3)$ & $98.9(14.7)\}$ & $59.9(6.1)$ & $147.2(13.2) \dagger$ \\
\hline 6 & $107.2(29.4)$ & $144.9(29.5)$ & $26.6(5.9)$ & $214.5(68.8)^{\star}$ \\
\hline 7 & $47.4(16.7)$ & $133.8(15.8) \dagger$ & $28.2(9.9)$ & $135.5(20.8) \dagger$ \\
\hline 8 & $76.6(5.4)$ & $70.9(11.4) \oint$ & $83.2(6.3)$ & $115.2(8.7) \dagger$ \\
\hline 9 & $184.0(28)$ & $137.5(16) 5$ & $92.7(6.7)$ & $164.0(18.4) \dagger$ \\
\hline
\end{tabular}

${ }^{\star} \mathrm{p}<0.05 ;+\mathrm{p}<0.01 ; \emptyset \mathrm{p}=$ NS. al found a progressive increase in pulmonary resistance and a prolongation of $\mathrm{T}_{\mathrm{e}}{ }^{9}$ Sanders and Moore ${ }^{10}$ measured the $R_{i}$ and $R_{e}$ of the two breaths preceding obstructive sleep-apnoea separately. Surprisingly, they found that both resistances were increased. ${ }^{10}$ As these changes were rapid and transient, these workers speculated that the mechanism responsible for the increase in $R_{e}$ was not related to an event in the lower airway, but rather to a pharyngeal narrowing during expiration caused by a dysfunction of the upper airway muscles. ${ }^{10}$ In the present study, the increase in $R_{e}$ and $T_{e}$ with various changes in $R_{i}$ and $T_{i}$ suggests that the increase in $R_{e}$ is not entirely a passive phenomenon caused by glossoptosis. The increase in $T_{e}$ and $R_{e}$ may be an active phenomenon modulated by an integrative neural mechanism.

During the ventilatory period characterised by severe glossoptotic pharyngeal obstruction we found: (1) a further decrease in the breathing rate; (2) increasing obstructed inspiratory efforts (equivalent of Muller's manoeuvre) often associated with stridor; (3) increasing obstructed expiratory efforts (equivalent of Valsalva's manoeuvre); and (4) retarded expiratory flow often associated with audible grunting. These findings are similar to those observed in children and adults with sleepapnoea syndrome. During the phase of obstructive sleep-apnoea, comparable studies in respiratory mechanics may detect increasing inspiratory efforts against a closed upper airway. ${ }^{9}{ }^{11} 12$ The obstructed inspiratory efforts are associated with an increase in expiratory resistance on account of positive gastric pressure ${ }^{11} 12$ or phasic expiratory activity of the abdominal muscles. ${ }^{13}$ The contractions of the expiratory muscles during sleep-apnoea may produce an audible expiratory flow. ${ }^{12}$ In addition, recording flow at high sensitivity, Sanders $e t$ al found a retarded expiratory flow which in adults with sleep-apnoea syndrome is associated with obstructed inspiratory flow. ${ }^{14}$ This expiratory flow pattern suggests that there may be a reduction in upper airway muscle activity during both inspiration and expiration. ${ }^{14}$ Alternatively, this retarded expiratory flow in adults with sleep-apnoea may indicate a prolonged active expiratory phase. ${ }^{15}$

The retarded expiratory flow pattern frequently observed in the present series of infants is not unlike that of active expiration 


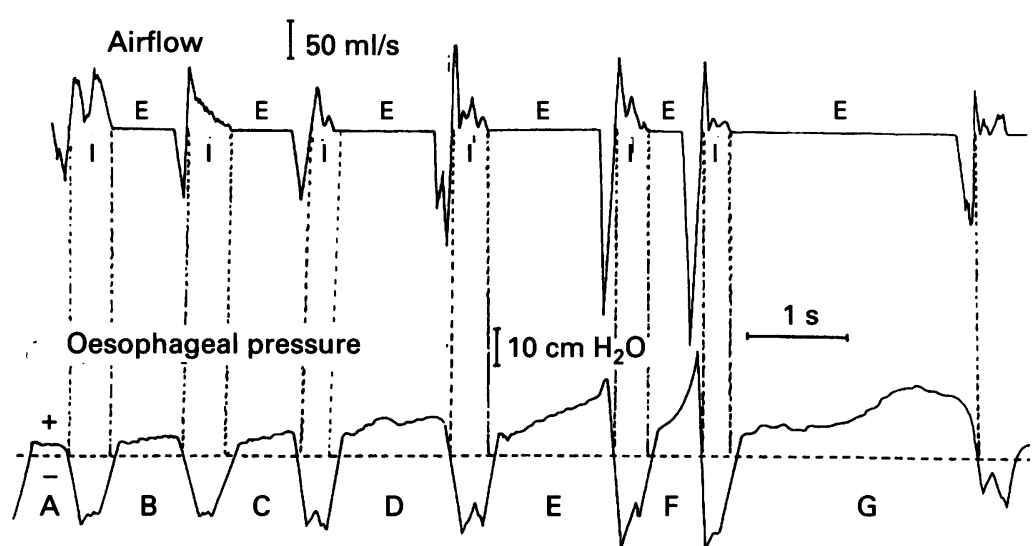

Figure 2 Representative ventilatory phase during severe glossoptotic pharyngeal obstruction. Note: (1) increasing inspiratory efforts matched with intrabreath obstruction of inspiratory flow; (2) increasing prolongation of expiratory phase (E); (3) increasing end expiratory positive oesophageal pressure swings matched with increasing expiratory flow late in expiratory phase; (4) the retarded expiratory flow may coincide with either a fall in positive end expiratory oesophageal pressure $(A-D, G)$ or with an increase in positive end expiratory oesophageal pressure $(E, F)$.

found in infants with hyaline membrane disease ${ }^{16}$ or even in normal infants with a subclinical grunt. ${ }^{17}{ }^{18}$ The present findings are consistent with the concept that infants with maturational upper airway instability may adopt the strategy of an active closure of the glottis to defend lung volume and lower airway patency by forcing gas into the peripheral airway during expiration. ${ }^{3}$ Indeed, many infants with maturational upper airway instability present, during phases of moderate respiratory distress, a greater degree of hypoxaemia than the degree of hypercapnia, and a marked alveolar-arterial oxygen difference when breathing air with high oxygen concentration. ${ }^{1}$ These blood gas derangements suggest that the upper airway obstruction gives rise to lower airway instability due to grossly hypoventilated and normoperfused areas of the lung liable to absorption collapse, right to left shunt, and hypoxaemia. ${ }^{1}$ In these infants, active braking of the expiratory flow during either partial or severe glossoptotic pharyngeal obstruction may be a very useful breathing strategy to avoid collapse of widespread areas of the lung and the consequent right to left shunt. Active braking of expiratory flow may be particularly important in infancy since a large intrapulmonary shunt may play a role in the pathogenesis of recurrent cyanotic attacks. ${ }^{19} 20$

The main difference between the expiratory flow pattern described in infants with clinical or subclinical grunting ${ }^{16-18}$ and what we observed during severe glossoptotic pharyngeal obstruction concerns the retarded expiratory flow. Sometimes, the latter may not necessarily coincide with a drop in $P_{\text {oes, }}$ as is usually found during grunting, but with an increasing end expiratory positive $P_{\text {oes }}$ (fig 2 ). These findings suggest that this pattern of expiration may be useful not only to increase the pressure inside the small airways, but also to relieve obstruction of the upper airway at the end of prolonged expiration.

Could the changes in airway mechanics observed here be related to the recurrent episodes presented by some infants with glossoptosis-apnoea syndrome and often diagnosed as 'bronchiolitis' or 'asthmatic attacks'?
Muller-Valsalva manoeuvres or the associated hypoxaemia in infants with chronic upper airway obstruction may cause, as in patients Nos 5 and 6, severe complications including pulmonary hypertension, non-cardiogenic pulmonary oedema, and cor pulmonale. ${ }^{121-23}$ The enlarged small pulmonary arteries and the peribronchial cuff of pulmonary oedema may compress the small airways, resulting in expiratory wheezing. An alternative explanation is that the dynamic collapse of the larger airways during forced expiration increases the resistance to airflow and produces expiratory wheezing. ${ }^{24}$

Another hypothesis is that a forced flow passing through a narrow glottic orifice may produce expiratory wheezing, thus simulating bronchospasm. ${ }^{24-26}$ The latter theory was first formulated in the attempt to explain clinical findings and results of lung function testing in patients with psychogenic wheeze. In these patients, like the infants in the present study, wheezing shows maximum intensity over the neck and may mimic bronchospasm over the chest. Rodenstein et al postulated that psychogenic wheezers have an emotional exaggeration of the normal physiological mechanism, which is adduction of the vocal cords during expiration. ${ }^{25} \mathrm{~A}$ similar hypothesis has recently been advanced to explain the pathophysiology of wheezing of the 'fat happy wheezers'. In these infants, Stecenko and Hutchinson have speculated that wheezing may be due to an overactive reflex in defence of lung volume or a delay in disappearance of the normal neonatal subclinical grunting pattern of expiration. ${ }^{27}$ The latter concept could explain why wheezing disappears spontaneously in many infants. ${ }^{27}$

In our patients, increased expiratory resistance during partial glossoptotic pharyngeal obstruction was associated with increased inspiratory resistance (table 3 ). In addition, during severe glossoptotic pharyngeal obstruction the expiratory grunt was always associated with a partial or totally obstructed inspiration. These findings suggest that the upper airway instability is the primary defect. The recurrent episodes diagnosed as 'bronchiolitis' or 'asthmatic attack' may therefore be substained by recurrent episodes of partial or severe glossoptotic pharyngeal obstruction triggered by an increase in respiratory efforts, as with crying, exertion, or respiratory infection. The ensuing lower airway instability brings about a grunting expiration which may simulate a bronchial obstruction.

Further studies are necessary to support the concept that glossoptotic pharyngeal obstruction should be added to the list of surgically correctable causes of upper andcentral airway obstruction responsible for wheezing (that is, laryngotracheomalacia, or extrinsic compression of the airway by vascular ring, mediastinal massses, or enlarged lymph nodes). If this view is proved correct, more severe cases of recurrent wheezing related to glossoptotic pharyngeal obstruction in infancy may require stabilisation of upper airway by glossopexy to relieve most of the symptoms 
and prevent additional respiratory pathology and sequelae.

1 Cozzi F, Pierro A. Glossoptosis-apnea syndrome in infancy. Pediatrics 1985;75:836-43.

2 Cozzi F, Steiner M, Rosati D, Madonna L, Colarossi G. Clinical manifestations of choanal atresia in infancy. $\mathcal{F}$ Pediatr Surg 1988;23:203-6.

3 Cozzi F, Myers NA, Madonna L, et al. Esophageal atresia, choanal atresia, and dysautonomia. F Pediatr Surg 1991; 26:548-52.

4 Cozzi F, Myers NA, Piacenti S, et al. Maturational dysautonomia and facial anomalies associated with esophageal atresia: support for neural crest involvement. $\mathcal{F}$ Pediatr Surg 1993;28:798-801.

5 Robin P. Glossoptosis due to atresia and hypotrophy of the mandible. Am $\mathcal{F}$ Dis Child 1934;48:541-7.

6 Roberts JL, Reed WR, Mathew O, Menon AA, Thach BT. Assessment of pharyngeal airway stability in normal and micrognathic infants. $\mathcal{f}$ Appl Physiol 1985;58:290-9.

7 Frank NR, Mead J, Perris BG. The mechanical behavior of the lungs in healthy elderly persons. $\mathcal{f}$ Clin Invest 1957;36:1680-7.

8 Polgar G, Kong GP. The nasal resistance of the newborn infants. F Pediatr 1965;67:557-67.

9 Martin RJ, Pennock BE, Orr WC, Sanders MH, Rogers RM. Respiratory mechanics and timing during sleep in RM. Respiratory mechanics and timing during sleep
occlusive sleep apnea. $₹$ Appl Physiol $1980 ; 48: 432-7$.

10 Sanders $\mathrm{MH}$, Moore SE. Inspiratory and expiratory partitioning of airway resistance during sleep in patients with sleep apnea. Am Rev Respir Dis 1983;127:554-8.

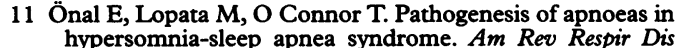
1982;125:167-74.

12 Remmers JE, De Groot WJ, Sauerland EK, Anch AM. Pathogenesis of upper airway occlusion during sleep. $\mathcal{F}$ Pathogenesis of upper airway

13 Jeffries B, Brouillette RT, Hunt CE. Electromyographic study of some accessory muscles of respiration in children with obstructive sleep apnea. Am Rev Respir Dis 1984; 129:696-702
14 Sanders $\mathrm{MH}$, Rogers RM, Pennock BE. Prolonged expiratory phase in sleep apnea. A unifying hypothesis. $\mathrm{Am}$ Rev Respir Dis 1985;131:401-8.

15 Johnson P. Prolonged expiratory apnoea and implication for control of breathing. Lancet 1985;ii:877-80

16 Harrison VC, de V Heese $\mathrm{H}$, Klein $M$. The significance of grunting in hyaline membrane disease. Pediatrics 1968; grunting in

17 Lindroth $M$, Johnson B, Ahlström $H$, Svenningrein NW. Pulmonary mechanics in early infancy. Subclinical grunting in low-birth-weight infants. Pediatr Res 1981;15:97984

18 Radvanyi-Bouvet MF, Monset-Couchard M, Morel-Kahn F, Vicente G, Dreyfus- Brisac C. Expiratory patterns during sleep in normal full-term and premature neonates. Biol Neonate 1982;41:74-84

19 Southall DP, Talber DG, Johnson P, et al. Prolonged expiratory apnoea: a disorder resulting in episodes of severe arterial hypoxaemia in infants and young children. Lancet 1985;ii:571-7.

20 Poets CF, Samuels MP, Southall DP. Potential role of intrapulmonary shunting in the genesis of hypoxemic episodes pulmonary shunting in the genesis of hypoxemic episodes

21 Luke MJ, Mehrizi A, Folger GM, Rowe RD. Chronic nasopharyngeal obstruction as a cause of cardiomegaly,
cor pulmonale and pulmonary edema. Pediatrics 1966; cor pulmo

22 Cox MA, Schiebler MD, Taylor WJ, Wheat MW, Krovetz LJ. Reversible pulmonary hypertension in a child with respiratory obstruction and cor pulmonale. $\mathcal{F}$ Pediatr 1965 ; 67:192-7.

23 Jeresaty RM, Huszar RJ, Basn S. Pierre Robin syndrome: cause of respiratory obstruction cor pulmonale, and pulmonary edema. Am $₹$ Dis Child 1969; 117:710-6.

24 Downing ET, Braman SS, Fox MJ, Corrao WM. Factitious asthma. Physiological approach to diagnosis. $\mathscr{f} A M A$ 1982; 248:2878-81.

25 Rodenstein DO, Francis C, Stanescu DC. Emotional laryngeal wheezing: a new syndrome. Am Rev Respir Dis 1983; geal wheezing

26 Christopher KL, Wood RP, Eckert RC, Blager FB, Raney RA, Sourada JF. Vocal-cord dysfunction presenting as asthma. N Engl f Med 1983;308:1566-70.

27 Stecenko AA, Hutchinson AA. Phascinating physiology. Am Rev Respir Dis 1991;144:1008-11. 